\title{
Studies of the oestrous cycle, oestrus and pregnancy in the koala (Phascolarctos cinereus)
}

\author{
S. D. Johnston ${ }^{1}$, M. R. McGowan ${ }^{1}$, P. O'Callaghan ${ }^{2}$, R. Cox ${ }^{3}$ and V. Nicolson ${ }^{2}$ \\ ${ }^{1}$ School of Veterinary Science and Animal Production, University of Queensland, 4072, Australia; ${ }^{2}$ Lone Pine Koala Sanctuary, \\ Jesmond Road, Fig Tree Pocket, 4069, Australia; and ${ }^{3}$ Bioquest Ltd, North Ryde, 2113, Australia
}

\begin{abstract}
As an integral part of the development of an artificial insemination programme in the captive koala, female reproductive physiology and behaviour were studied. The oestrous cycle in non-mated and mated koalas was characterized by means of behavioural oestrus, morphology of external genitalia and changes in the peripheral plasma concentrations of oestradiol and progestogen. The mean ( \pm SEM) duration of the non-mated oestrous cycle and duration of oestrus in 12 koalas was $32.9 \pm 1.1(n=22)$ and $10.3 \pm 0.9(n=24)$ days, respectively. Although the commencement of oestrous behaviour was associated with increasing or high concentrations of oestradiol, there were no consistent changes in the morphology or appearance of the clitoris, pericloacal region, pouch or mammary teats that could be used to characterize the non-mated cycle. As progestogen concentrations remained at basal values throughout the interoestrous period, non-mated cycles were considered non-luteal and presumed anovulatory. After mating of the 12 koalas, six females gave birth with a mean ( \pm SEM) gestation of $34.8 \pm 0.3$ days, whereas the remaining six non-parturient females returned to oestrus $49.5 \pm 1.0$ days later. After mating, oestrous behaviour ceased and the progestogen profile showed a significant increase in both pregnant and non-parturient females, indicating that a luteal phase had been induced by the physical act of mating. Progestogen concentrations throughout the luteal phase of the pregnant females were significantly higher than those of non-parturient females. Parturition was associated with a decreasing concentration of progestogen, which was increased above that of basal concentrations until 7 days post partum.
\end{abstract}

\section{Introduction}

Despite the popularity of the koala both in Australia and overseas, knowledge of the reproductive physiology of this species is incomplete. For example, although it is generally accepted that the koala is seasonally polyoestrous, estimates of the duration of the oestrous cycle (Briese, 1970; Handasyde, 1986; Brown, 1987; Thompson, 1987; Johnston, 1994) and oestrus (Smith, 1980; Thompson, 1987) vary markedly from 15 to 57 days, and $2.5 \mathrm{~h}$ to 14 days, respectively. There is only limited information describing the reproductive endocrinology of the oestrous cycle (Brown, 1987; Handasyde et al., 1990) and conflicting reports on the timing of sexual maturity of wild and captive koala populations (Tyndale-Biscoe and Renfree, 1987; O'Callaghan, 1996). Furthermore, some authors (Brown, 1987; Handasyde et al., 1990; Johnston, 1994) have suggested that ovulation in this species may be induced by coital stimulation, a phenomenon that, to date, has not been examined in detail and which would be unique among marsupials.

Received 20 September 1999.
As part of the development of an artificial insemination programme in this species, the aim of the present study was to characterize the oestrous cycle of non-mated and mated koalas. The duration of the non-mated and mated oestrous cycle was determined by observations of behavioural oestrus, and the relationships between oestrus, changes in the appearance of external genitalia and peripheral plasma concentrations of progestogen and oestradiol were investigated.

\section{Materials and Methods}

\section{Animals}

Female koalas used in this study were housed together in a single large $(12 \mathrm{~m} \times 4 \mathrm{~m} \times 3 \mathrm{~m})$ naturally lit enclosure, located at Lone Pine Koala Sanctuary (LPKS), Brisbane, Australia (Blanshard, 1994). All 12 females were sexually mature, ranged in age from 3 to 9 years, had no history of reproductive disease and remained healthy throughout the study period. All procedures carried out in this study were approved by LPKS and University of Queensland animal ethics committees. 


\section{Detection of behavioural oestrus}

Oestrous behaviour in the koala has been described by Smith (1980), Blanshard (1994) and reviewed by Johnston (1999). In brief, the most conspicuous oestrous behaviour is 'jerking' or convulsive behaviour, which resembles hiccoughing. Females in oestrus may also show what has been termed homosexual or pseudo-male mounting behaviour (Smith, 1980) whereby they appear to mimic male copulatory behaviour. A female will also characteristically bellow when she is in oestrus, display increased activity and appear restless.

In this study, oestrus was detected using the method described by Blanshard (1994). A 'teaser' male was brought in and placed on the floor of the female enclosure. The male was allowed to scent mark objects with his sternal gland, bellow and urinate. Introduction of the male immediately evoked oestrous behaviour in those females that were sexually receptive. Females were considered to be in oestrus if they displayed one or all of the oestrous behaviours described above. Oestrous detection was conducted between 16:00 $\mathrm{h}$ and 17:00 $\mathrm{h}$ each day from September 1996 to the completion of the study in March 1997. The 'teaser' males were regularly rotated to avoid the possibility of females or males becoming habituated to the oestrous detection procedure. The male was also presented individually to each female to ensure that all females in the enclosure had equal visual and physical access to the 'teaser'; however, no direct contact between males and females occurred.

Preliminary observations revealed that oestrous females appeared to mount other oestrous females preferentially to non-oestrous females. Observations of those females who were mounted by oestrous females were recorded between September and November 1996 to confirm the preliminary observations.

\section{Examination of the external genitalia}

Brown (1987) reported that during oestrus, the clitoris of the koala becomes hyperaemic and the hairless pericloacal region becomes enlarged and oedematous. The degree of clitoral hyperaemia was determined qualitatively in this study as engorged or not engorged, whereas oedematous changes in the appearance of the pericloacal region were evaluated subjectively as either enlarged or not enlarged. Pouch condition throughout the oestrous cycle was classified subjectively as clean or dirty, dry or moist. The presence or absence of papillae on the terminal portion of the mammary teats was also noted (Sharman and Calaby, 1964).

\section{Blood collection}

Koala handling and blood collection procedures were as described Blanshard (1994). Samples (2 ml) of blood were collected sequentially from the cephalic vein using a $12 \mathrm{~mm}$ 25 gauge needle attached to a $0.5 \mathrm{~mm} \times 9.5 \mathrm{~mm}$ winged infusion set (Terumo, Tokyo) and a $3 \mathrm{ml}$ syringe. The sample was divided equally into $1 \mathrm{ml}$ heparinized tubes (Becton Dickinson, NJ) and centrifuged at $1600 \mathrm{~g}$ for $10 \mathrm{~min}$. Plasma samples were stored in a $-20^{\circ} \mathrm{C}$ freezer. As the koalas at LPKS are handled regularly by staff, the collection of blood samples resulted in little or no distress (minor struggling) to the animal.

\section{Oestradiol and progestogen analysis}

The concentration of oestradiol (1,3,5(10)-estratriene-3,17(-diol)) and progesterone (4-pregnen-3,20-dione) in the peripheral blood of koalas was assayed directly from plasma using DELFIA ${ }^{\circledR}$ (solid-phase fluroimmunoassay) oestradiol and progesterone kits (Wallac, Turku). The fluorescence was measured using a time-resolved fluorometer (Model 1230; Arcus LKB Wallac). Both assays were validated for koala plasma. Recovery of steroid added to koala plasma was $106 \pm 8.5 \%(\mathrm{SD})(n=15)$ for oestradiol in the range $24.5-$ $81.7 \mathrm{pg} \mathrm{ml}^{-1}$ and $99 \pm 11.5 \%(\mathrm{SD})(n=22)$ for progesterone in the range $0.4-22.6 \mathrm{ng} \mathrm{ml}^{-1}$. Interassay coefficients of variation for oestradiol were $7.8 \%(n=5)$ and $7.3 \% \quad(n=10)$ at $24.5 \mathrm{pg} \mathrm{ml}^{-1}$ and $81.7 \mathrm{pg} \mathrm{ml}^{-1}$, respectively. For progesterone, estimated interassay coefficients of variation were $15.1 \%$ $(n=9)$ at $1.1 \mathrm{ng} \mathrm{ml}^{-1}, 13.8 \%(n=8)$ at $3.8 \mathrm{ng} \mathrm{ml}^{-1}$ and $8.1 \%$ $(n=10)$ at $15.7 \mathrm{ng} \mathrm{ml}^{-1}$. The detection limit of the DELFIA ${ }^{\circledR}$ assays $(2 \times$ SD calculated from measurement values of zero standards) was $8.7 \mathrm{pg} \mathrm{ml}^{-1}(n=24)$ for oestradiol and $0.1 \mathrm{ng} \mathrm{ml}^{-1}(n=18)$ for progesterone. All assays using plasma were carried out in duplicate except samples for the progesterone assay taken during the interoestrous period of the first two non-mated cycles.

The only appreciable crossreactivity with the progesterone antisera was that of $5 \alpha$-dihydroprogesterone (45.0\%) (see Table 1). McDonald and Than (unpublished data in Handasyde et al., 1990) used HPLC to identify steroids in peripheral blood plasma of the female koala. These authors identified oestradiol and progesterone as the major reproductive steroids, as well as high concentrations of pregnenelone and 20-hydroxyprogesterone and minor concentrations of oestrone, oestriol and testosterone, but did not record the presence of $5 \beta$-dihydroprogesterone. $5 \beta$ dihydroprogesterone can be derived directly from progesterone in the first step of its metabolism. Therefore, even if this compound is present in koala plasma in appreciable amounts, relative to progesterone, it would closely parallel changes in progesterone concentration. However, as the progesterone assay used in the present study crossreacted significantly with $5 \beta$-dihydroprogesterone, the term 'progestogen' will be used in recognition that the DELFIA $^{\circledR}$ assay system may overestimate the absolute value of progesterone in the koala plasma. There was only limited crossreactivity with other hormones using oestradiol antisera (Table 1).

\section{Experiment 1: characterization of the oestrous cycle in non- mated koalas}

In Expt 1, all 12 koalas were monitored each day for signs of behavioural oestrus throughout two successive nonmated oestrous cycles. The duration of the oestrous cycle 
Table 1. Crossreactivity of oestradiol and progesterone assays

\begin{tabular}{lc}
\hline Substance & Crossreactivity (\%) \\
\hline Oestradiol assay at 50\% inhibition & \\
Oestrone & 0.75 \\
Oestrone-3-sulphate & 0.02 \\
Oestriol & 0.40 \\
2-Hydroxyoestradiol & 0.02 \\
16-Oxoestradiol & 0.90 \\
16-Hydroxyoestrone & 0.04 \\
Oestradiol-3-sulphate, sodium salt & 0.14 \\
Oestradiol-3-glucuronide, sodium salt & 0.55 \\
& \\
Progesterone assay at 50\% inhibition & \\
5 $\beta$-Dihydroprogesterone & 45.0 \\
5 $\alpha$-Dihydroprogesterone & 6.5 \\
Corticosterone & 1.2 \\
17 $\alpha$-Hydroxyprogesterone & 0.63 \\
Pregnanolone & 0.40 \\
20 $\beta$-Dihydroprogesterone & 0.14 \\
Pregnenolone & 0.11 \\
11-Deoxycortisol & 0.06 \\
11-Deoxycorticosterone & 0.05 \\
20 $\alpha$-Dihydroprogesterone & 0.05 \\
\hline
\end{tabular}

was defined as the time interval between the first day of two successive periods of overt oestrous behaviour. The first day of behavioural oestrus was designated as day 0. Blood samples were collected from all 12 koalas throughout two non-mated oestrous cycles, typically on days $0,2,4,7,14,21$, 28,32 and 34 of the oestrous cycle. Changes in the morphology of the external genitalia during this period were recorded from only six koalas, typically on days $0,1,2,4,7$, $10,14,18,21,24,28,32$ and 34 . For oestrous cycles that were $>34$ days, blood sampling and observation of the genitalia were carried out every third day thereafter until oestrus.

\section{Experiment 2: characterization of the oestrous cycle in mated koalas}

At their third oestrus, each female was mated. The 12 koalas were randomly assigned to two groups to investigate whether pregnancy was influenced by the stage of oestrus during which the female was mated. On the basis of the average duration of the previous two oestrous periods for each koala, one group was mated on the day during the period of behavioural oestrus equivalent to approximately one-quarter of the duration of their average oestrus; the other group was mated at approximately one-half the duration of their average oestrus. For example, in the case of two females with an average oestrus of 8 days, one was mated on day 2 , the other on day 4 . The pregnancy rates of both groups were compared by chi-squared analysis (Feldman and Gagnon, 1991). Eight of the 12 copulations were recorded individually on video-tape for event analysis. The number of thrusts and the period of thrusting and ejaculation were determined.

After mating, all 12 koalas were monitored for behavioural
Table 2. Duration of the oestrous cycle and oestrus for two non-mated cycles in the koala

\begin{tabular}{lccccc}
\hline & \multicolumn{2}{c}{$\begin{array}{c}\text { Duration of the } \\
\text { oestrous cycle (days) }\end{array}$} & & \multicolumn{2}{c}{$\begin{array}{c}\text { Duration of } \\
\text { oestrus (days) }\end{array}$} \\
\cline { 2 - 3 } \cline { 5 - 6 } Koala no. & Cycle 1 & Cycle 2 & & Cycle 1 & Cycle 2 \\
\hline F1 & 36 & 39 & & 10 & 13 \\
F2 & 38 & 48 & & 14 & 19 \\
F3 & 34 & 32 & & 9 & 11 \\
F4 & 31 & 25 & & 9 & 7 \\
F5 & 28 & 25 & & 11 & 11 \\
F6 & 29 & 37 & & 10 & 15 \\
F7 & 36 & 39 & & 15 & 14 \\
F8 & 33 & 33 & & 11 & 15 \\
F9 & 32 & 32 & & 8 & 10 \\
F10 & 26 & 28 & & 10 & 12 \\
F11 & 28 & 34 & & 4 & 4 \\
F12 & NA & NA & & 5 & 0 \\
Mean \pm SEM & $32.9 \pm 1.1(n=22)$ & & $10.3 \pm 0.9(n=24)$ \\
\hline
\end{tabular}

NA: not available.

*Animal apparently had a 'silent' oestrus.

oestrus each day and external genitalia were examined on days $0,2,4,5,7,8,10,11,13,18,21,24,28,30,32$ and 34 after mating. In this case, day 0 corresponded to the day of mating. Blood samples were collected on days $0,2,5,8,11$, $14,18,21,28,32$ and 34 after mating. Of the 12 koalas that were mated, six produced pouch young and six returned to a fourth oestrus. Detection of oestrus and blood sampling were continued up to 40 days post partum in the koalas that gave birth (days 37, 41, 44, 49, 54, 59, 64, 69 and 74 after mating) or until a fourth oestrus was detected in the females that failed to give birth (days 37, 41, 44 and then every fifth day until the onset of the fourth oestrus).

The duration of the oestrous cycle in mated and nonmated females was compared using a Student's unpaired $t$ test (Feldman and Gagnon, 1991). Changes in oestradiol and progestogen concentrations during the luteal phase between pregnant animals $(n=6)$ and those that returned to oestrus (non-parturient) $(n=6)$ were compared using a nonrepeated measures two-way ANOVA (Feldman and Gagnon, 1991).

\section{Results}

\section{Non-mated oestrous cycles}

The mean ( \pm SEM) duration of 22 non-mated cycles for 11 koalas was $32.9 \pm 1.1$ days; one female (F12) failed to display a second oestrus. There was considerable variation in the duration of the oestrous cycle of individual koalas, ranging from 25 to 45 days. In some koalas, there was also substantial variation between the duration of consecutive cycles for the same individual (Table 2$)$. The mean ( \pm SEM) duration of oestrus for 24 non-mated cycles was $10.3 \pm 0.9$ days. There was also substantial variation in the duration of behavioural oestrus ranging from 0 to 19 days (Table 2). 


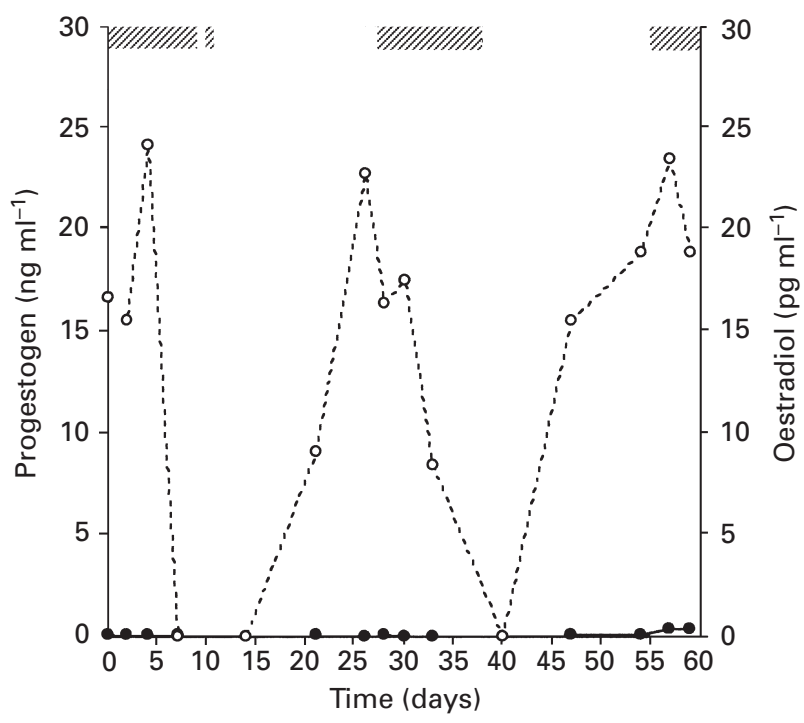

Fig. 1. Typical oestradiol $\left(\mathrm{pg} \mathrm{m} \mathrm{ml}^{-1}\right)$ and progestogen $\left(\mathrm{ng} \mathrm{ml}^{-1}\right)$ profiles of two consecutive non-mated oestrous cycles for female koala F10: 四, behavioural oestrus; - - progesterone concentration $\left(\mathrm{ng} \mathrm{ml}^{-1}\right)$; ---○---, oestradiol concentration $\left(\mathrm{pg} \mathrm{ml}^{-1}\right)$.

Throughout the two non-mated cycles of all 12 koalas, there was no consistent pattern of change in the appearance of the clitoris, pericloacal region, teats or pouch that could be used to characterize the oestrous cycle.

The typical relationship between oestradiol concentration and oestrous behaviour throughout two non-mated cycles is shown (Fig. 1). Although the onset of oestrus was typically associated with increasing or high oestradiol concentrations, on 37 of 41 occasions (90\%), oestradiol concentration did not remain high throughout the entire duration of behavioural oestrus. The mean ( \pm SEM) peak concentration of oestradiol observed during three consecutive occurrences of oestrus from a total of 12 non-mated koalas $(n=35)$ was $27.3 \pm 1.8 \mathrm{pg} \mathrm{ml}^{-1}$ (range 8.7-45.2 $\mathrm{pg} \mathrm{ml}^{-1}$ ). Although the typical oestradiol profile is presented (Fig. 1), it should be noted that there was considerable variation in the oestradiol profile of individual animals: some koalas (4/12) showed slightly increased concentrations of oestradiol during the interoestrous period but with no accompanying oestrous behaviour.

The typical plasma progestogen profile throughout two non-mated oestrous cycles in a female koala is also presented (Fig. 1). The mean ( \pm SEM) progestogen concentration of 173 samples from the non-mated cycles of 12 koalas was $0.38 \pm 0.03 \mathrm{ng} \mathrm{ml}^{-1}$. The progestogen concentration remained consistently low throughout the non-mated cycles and did not exceed $2 \mathrm{ng} \mathrm{ml}^{-1}$.

\section{Observations of copulatory behaviour}

For the males in which copulatory behaviour was observed in detail $(n=8)$, the mean ( \pm SEM) number of penile thrusts and duration of thrusting were $33.9 \pm 5.8$ and
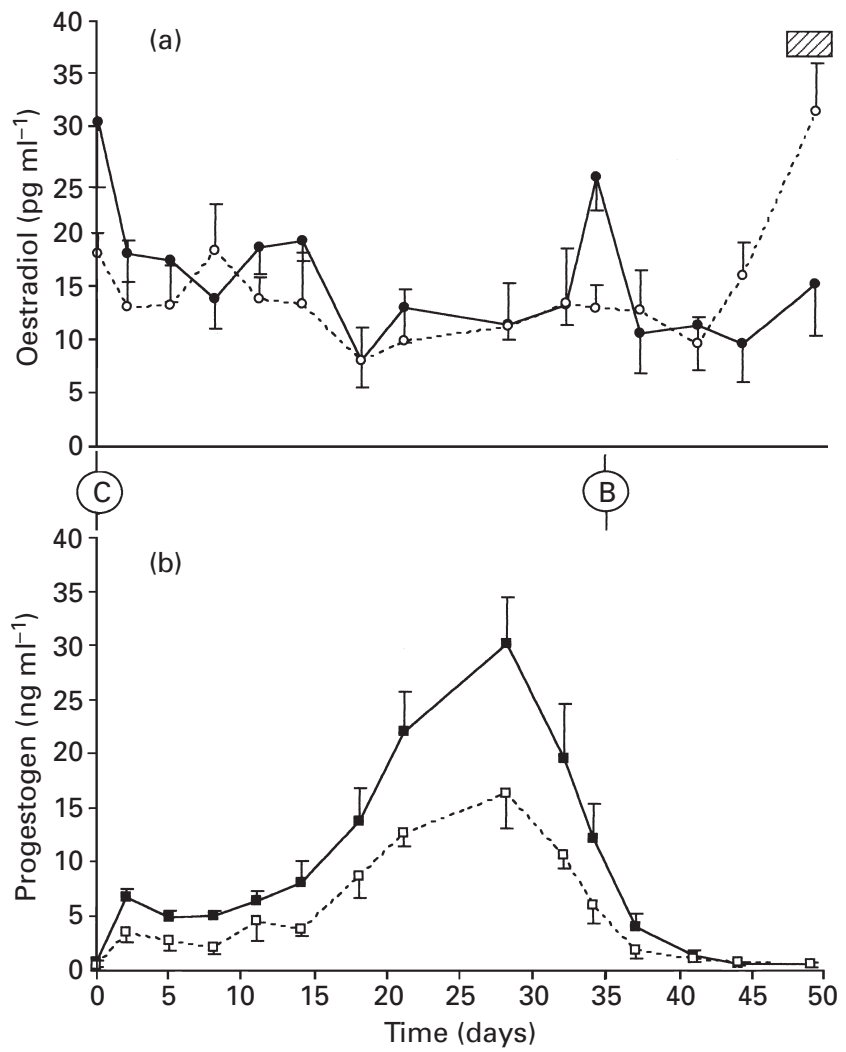

Fig. 2. Mean ( \pm SEM) concentration of (a) oestradiol and (b) progestogen up to 49 days after mating in six pregnant and six nonparturient koalas. C: day of mating; B: day of parturition; , behavioural oestrus; - - , oestradiol concentration $\left(\mathrm{pg} \mathrm{ml}^{-1}\right)$ of pregnant females; ---○---, oestradiol concentration $\left(\mathrm{pg} \mathrm{ml}^{-1}\right)$ of nonparturient females; - - , progestogen concentration $\left(\mathrm{ng} \mathrm{ml}^{-1}\right)$ of pregnant females; --- $\square---$, progestogen concentration $\left(\mathrm{ng} \mathrm{ml}^{-1}\right)$ of non-parturient females.

$28.1 \pm 4.0 \mathrm{~s}$, respectively. However, there was a wide range in the number of thrusts performed by each male (range 5-52) and the duration of thrusting (16-50 s). The mean ( \pm SEM) duration of ejaculation was $21.0 \pm 5.2 \mathrm{~s}$. One male thrusted his penis into the urogenital sinus of the female (F4) only five times over a $50 \mathrm{~s}$ period and then ejaculated outside the female. Another male thrusted normally but withdrew his penis from the urogenital sinus of the female (F6) after only 4 $\mathrm{s}$ of ejaculatory behaviour. Females F4 and F6 failed to give birth. A dried semen plug was observed around the cloacal region of all ten females that received normal male copulatory behaviour. Females F3 and F8 had a serous blood stained discharge from the urogenital sinus during and immediately after mating.

\section{Pregnancy and the postpartum period}

The duration of pregnancy for six koalas was $34.8 \pm 0.3$ days (mean $\pm \mathrm{SEM}$; range 34-36 days). There was no difference in the pregnancy rate of those koalas mated on a 
day equivalent to one-quarter of the average duration of oestrus (2/5) compared with koalas mated on a day equivalent to one-half of the average duration of oestrus $(4 / 7)$.

The mean oestradiol profile in the peripheral plasma of six pregnant koalas from the day of mating to pregnancy and the peripartum period up to 49 days after mating is shown (Fig. 2). These results show that the oestradiol concentration varied significantly throughout this period $(F=2.48$; $\mathrm{df}=14,84 ; P<0.01)$ and there were peaks during oestrus and immediately before birth. Oestrous behaviour was not apparent throughout pregnancy or the peripartum period up to 70 days after mating (results $>49$ days not shown). Oestradiol was also generally at its lowest concentration during the period of peak progestogen concentration.

The mean progestogen profile in the peripheral plasma of six pregnant koalas throughout pregnancy and the peripartum period up to 49 days after mating is also shown (Fig. 2). The results show that progestogen concentration varied significantly throughout this period $(F=14.94$; $\mathrm{df}=14,86 ; P<0.01)$. All koalas showed a similar pattern of increased progestogen concentration after mating. At day 2 after mating there was a marked increase in progestogen concentration to approximately $7 \mathrm{ng} \mathrm{ml}^{-1}$ followed by a decrease to $5 \mathrm{ng} \mathrm{ml}^{-1}$ on day 5 . At day 11 after mating, the progestogen concentration began to increase again, and by day 28 after mating had reached a peak of about $30 \mathrm{ng} \mathrm{ml}^{-1}$. Subsequently, there was a progressive decrease in progestogen output such that at day 32 after mating the concentration had decreased to approximately $18 \mathrm{ng} \mathrm{ml}^{-1}$. The progestogen concentration was still high within 1 day of parturition $\left(10 \mathrm{ng} \mathrm{ml}^{-1}\right)$ and even at day 3 post partum remained slightly increased at $4 \mathrm{ng} \mathrm{ml}^{-1}$. The concentration of progestogen decreased to basal values $\left(<1 \mathrm{ng} \mathrm{ml}^{-1}\right)$ at day 7 after birth.

\section{Mated non-parturient oestrous cycle}

After mating, the interoestrous interval of females that did not produce pouch young was $52.5 \pm 0.8$ days $(n=6)$. This interval was significantly longer $(t=8.32 ; P<0.01)$ and less variable (range 51-56 days) than that of non-mated oestrous cycles. The time from the day of mating to expression of the next oestrus in the females that did not give birth was $49.5 \pm 1.0$ days (range 47-53 days): this value represents an estimate of the duration of both the luteal and subsequent follicular phase in the koala.

The mean oestradiol profile in the plasma of six koalas that were mated but did not produce pouch young is shown (Fig. 2). The concentration of oestradiol varied significantly ( $F=1.87 ; \mathrm{df}=14,89 ; \quad P<0.05)$ throughout the luteal and subsequent follicular phase. The oestradiol peak associated with oestrus on the day of mating was not as pronounced as that which occurred when the females returned to oestrus approximately 49 days after mating $(t=-2.68 ; \mathrm{df}=10$; $P<0.02)$. The mean $( \pm$ SEM) peak oestradiol concentration during this oestrus was $33.9 \pm 3.8 \mathrm{pg} \mathrm{ml}^{-1}$. As with pregnant koalas, the concentration of oestradiol during the luteal phase of mated non-parturient koalas was generally lower, coincident with the high progestogen concentration during this period.

The significant changes $(F=13.4 ; \mathrm{df}=14,89 ; P<0.01)$ in the mean $( \pm$ SEM) progestogen profile of six mated but nonparturient koalas during the luteal phase and the period leading up to expression of the subsequent oestrus is summarized (Fig. 2).

\section{Comparison of oestradiol profiles of pregnant and non- parturient koalas}

A two-way ANOVA of the oestradiol profiles throughout the luteal phase and subsequent period up to 49 days after mating revealed that although the profiles of both pregnant and non-parturient animals varied significantly throughout this period, there was no statistical difference in oestradiol concentration between the profiles with respect to pregnancy status. There were apparent differences between pregnant and non-parturient animals in the concentration of oestradiol at specific times: however this effect was not significant on the day of mating or in the period immediately before birth, but was significant in the period when nonparturient females returned to oestrus $(t=-2.46 ; \mathrm{df}=10$; $P<0.05)$.

\section{Comparison of progestogen profiles of pregnant and non- parturient koalas}

Results of a two-way ANOVA revealed that progestogen concentrations of non-parturient koalas were significantly lower than those of pregnant koalas $(F=32.5 ; \mathrm{df}=1,14$; $P<0.01)$ and that progestogen concentration varied throughout mated cycles $(F=27.3$; df $=14,175 ; P<0.01)$. The interaction between parturition status and the concentration of progestogen throughout the luteal phase was also significant $(F=2.57 ; \mathrm{df}=1,14 ; P<0.01)$. Although it was not possible to assess whether the non-parturient condition was a consequence of fertilization failure or embryonic mortality, it should be noted that the copulations of F4 and F6 were potentially 'sterile' as although the respective males showed thrusting behaviour, they did not appear to ejaculate into the female.

\section{Changes in the external genitalia of the female after mating}

After copulation, there were no consistent patterns of change in the appearance of the clitoris, teats or pericloacal region. However, there were detectable changes in the secretory activity of the pouch skin. Although the inner lining of the pouch of all 12 females was dry at oestrus and immediately after mating, pouch secretion appeared to increase progressively during the later stages of the luteal phase in both pregnant and mated but non-parturient females. Both pregnant and non-parturient females also resented pouch inspection performed from 34 days after mating. 


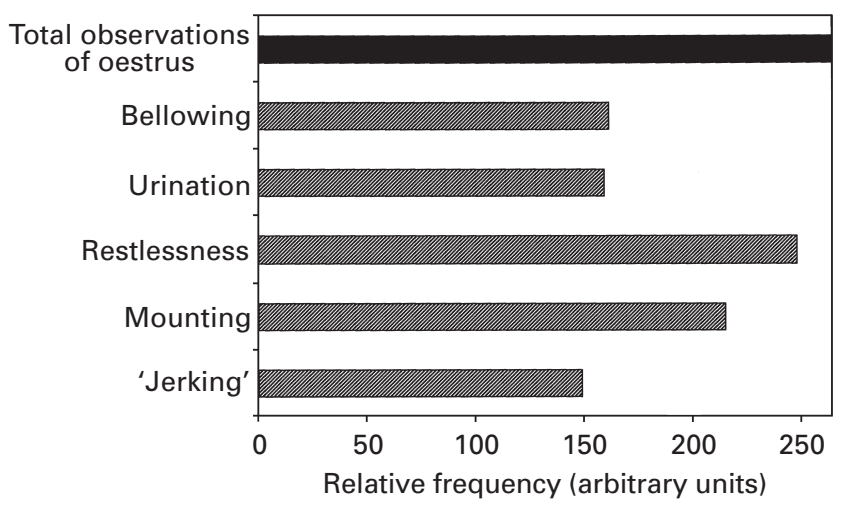

Fig. 3. The relative frequencies of oestrous behaviours of 12 female koalas over two oestrous periods.

\section{Observations of behavioural oestrus}

The relative frequencies of the various types of oestrous behaviour from 254 individual observations of oestrus throughout the study period are presented (Fig. 3). Restlessness and pseudo-male mounting behaviours were the most frequently observed oestrous behaviours. There was also substantial individual variability in the expression of the range of oestrous behaviours; for example F7 displayed oestrus for a total of 30 days but bellowed on only six occasions, whereas F10 displayed oestrus for a total of 29 days and bellowed on 26 occasions. There were also numerous occasions on which oestrous behaviours were not consistently or continuously displayed throughout the duration of oestrus. One female (F12) initially displayed oestrus at the start of the study period, but then did not show any signs of oestrus until 70 days later. It is possible that this female had a silent oestrus that was not detected.

From a total of 192 observations of pseudo-male mounting behaviour, oestrous females were found to have mounted other oestrous females on 140 occasions (71.8\%), a significantly greater number than if oestrous females had chosen to mount females at random $\left(\chi^{2}=47.3 ; P<0.01\right)$.

\section{Discussion}

The results of the present study confirm the observations of Brown (1987), Handasyde et al. (1990) and Johnston (1994) that if koalas are prevented from mating, they are polyoestrous and have an interoestrous interval ranging between 25 and 48 days. The finding that increasing or high plasma oestradiol concentrations in the female koala coincided with, or preceded, periods of behavioural oestrus is consistent with the study of Brown (1987). The concentrations of oestradiol detected in the plasma of oestrous koalas during non-mated cycles in the present study were approximately half the magnitude of those reported by Brown (1987), but similar to those reported by Handasyde et al. (1990). Peripheral plasma oestradiol concentrations of most female koalas decreased before cessation of oestrous behaviour, indicating that although high concentrations of oestradiol may be important for initiating oestrus in koalas, other factors are involved in progression of oestrus. A high oestradiol concentration is associated with oestrous behaviour in other marsupials including the brushtail possum (Trichosurus vulpecula; Curlewis et al., 1985) and tammar wallaby (Macropus eugenii; Shaw and Renfree, 1984; Harder et al., 1985), but is in contrast to the findings of Harder and Fleming (1981) who reported high concentrations of oestradiol in the Virginian opossum (Didelphis virginiana; $24 \mathrm{pg} \mathrm{ml}^{-1}$ ) at 2-4 days before oestrus. In ewes, oestrous expression requires priming with progesterone (Short, 1982), whereas in rodents it can be induced artificially by administration of oestrogen followed by progesterone (Herbert, 1972). However, koalas appear to be similar to induced ovulators like the domestic cat; oestrus is associated with high oestradiol concentrations but is preceded by, and co-incident, only with basal concentrations of progestogen.

Hormone profiles of some individual koalas observed in the present study showed moderately high oestradiol concentrations during the interoestrous period of non-mated cycles. It is possible that oestradiol secreted during this period is produced from a second wave of follicles that develop as the dominant follicle of the previous follicular wave starts to regress. Although waves of follicular growth in the ovary have been demonstrated in a number of domestic animal species (Adams, 1998), this concept has received little if any attention in studies of ovarian follicular dynamics in marsupials.

For all 12 koalas in this study, the progestogen concentration in the peripheral circulation throughout two non-mated oestrous cycles remained at basal values $\left(0.38 \pm 0.03 \mathrm{ng} \mathrm{ml}^{-1}\right)$, indicating that a luteal phase did not occur during this period. It should be noted that throughout these two oestrous cycles females were regularly exposed to males displaying typical pre-mating behaviour (bellowing, urination, scent marking), but there was no evidence that this exposure resulted in the induction of a luteal phase. As originally proposed but not demonstrated by McDonald and Lithgow (unpublished observation in Tyndale-Biscoe and Renfree, 1987), it appears that non-mated oestrous cycles in the koala may be shortened anovulatory cycles $(32.9 \pm 1.1$ days), representative of at least one period of follicular growth and atresia, but no luteal phase. Such patterns of follicular growth and atresia have been reported in the nonmated cycles of induced ovulators such as rabbits (Hammond, 1925), Camelus dromedarius (Musa et al., 1993) and llamas (Lama glama; Bravo et al., 1990; Adams, 1992). Shortened anovulatory cycles have also been reported for the marsupial species D. virginiana (Hartman, 1923; MartinezEsteve, 1937) and the mouse opossum (Marmosa robinsonii; Godfrey, 1975), but without accompanying evidence of progesterone profiles or histological evidence that ovulation occurred.

Brown (1987) has reported that the clitoris of the koala became hyperaemic and the pericloacal region enlarged during oestrus. However, in the present study no reliable or consistent changes in the appearance of the clitoris or pericloacal region were observed in either non-mated or mated oestrous cycles, and consequently monitoring the 
appearance of these structures was not considered useful for the determination of the stage of the oestrous cycle or predicting parturition. There was also no consistent pattern of change in the condition of the pouch or morphology of the teats during non-mated cycles.

After mating, the oestradiol concentrations of both nonparturient and pregnant females decreased progressively whereas progestogen concentrations increased; this finding is consistent with the ovulation of the dominant follicle and subsequent formation of the corpus luteum. During periods of peak progestogen secretion the concentration of oestradiol remained relatively low, only increasing in pregnant animals immediately before parturition and co-incident with the return of oestrus in non-parturient females. The oestradiol peak before parturition was not associated with oestrous behaviour and thus its functional significance remains unclear. However, the absence of a similar oestradiol peak in non-parturient animals indicates that it may be associated with onset of parturition, or alternatively is simply the result of the continuance of follicular development and production of a dominant follicle throughout the luteal phase. This finding warrants further investigation.

Comparative data of oestradiol profiles throughout marsupial pregnancy and the non-pregnant cycle are limited to three species. Except at oestrus and at about day 5 of the oestrous cycle, plasma oestradiol concentrations in M. eugenii are low to undetectable (Hinds et al., 1996). A similar situation is observed in T. vulpecula, in which oestradiol has been detected only at the time of oestrus (Curlewis et al., 1985). Harder and Fleming (1981) reported widely fluctuating oestradiol concentrations in the luteal phase of both pregnant and non-pregnant D. virginiana. Although Harder and Fleming (1981) ascribed no functional significance to this phenomenon, it is possible that variable oestradiol secretion in $D$. virginiana is related to different stages of follicular development, which apparently continues throughout the luteal phase. Although not presented specifically in the results section of this study, there was also evidence of significant fluctuations in oestradiol concentration throughout the post-coital period of individual koalas, which is not apparent from the presentation of mean values.

After mating, the peripheral progestogen concentration measured in all 12 koalas increased significantly. This is the first report of a mating-induced luteal phase in a marsupial. As 6 of 12 koalas gave birth approximately 35 days after mating, there is strong secondary evidence to indicate that ovulation in the koala is also induced by mating. Although studies of M. domestica (Fadem and Ravye, 1985), the woolly opossum (Caluromys philander; Perret and Ben M'Barek, 1991), as well as the brush-tailed bettong (Bettongia penicillata; Smith, 1992; Hinds and Smith, 1992; Smith, 1994) and T. vulpecula (Crawford et al., 1997) have indicated that in these species oestrus may be induced by the presence or introduction of the male to the female, it is uncertain whether ovulation and the subsequent luteal phase is actually triggered by mating. Results from the present study indicate that induction of the luteal phase (and most probably ovulation) in koalas requires the physical act of mating rather than just exposure to the male. This contention is supported by the fact that in the present study all female koalas that were exposed to 'teaser' males but had no physical contact, showed no evidence of a luteal phase. It is proposed that, in koalas, the luteal phase of the oestrous cycle (including ovulation) may involve the triggering of a copuloceptive reflex similar to that described in the domestic cat (Felis catus; Banks, 1986) and rabbit (Ramirez and Lin Soufi, 1994). Studies confirming the presence of matinginduced ovulation in the koala are the subject of an adjoining paper in this volume (Johnston et al., 2000). In one female koala in the present study, a luteal phase was induced after only five penile thrusts into the urogenital sinus, indicating that there may be considerable individual variability in the level of stimulus required to trigger a luteal phase. Copulation in the koala is quite a vigorous process; on two separate occasions immediately after mating, females showed evidence of trauma (slight bloody discharge from the cloaca) to their urogenital sinus.

An oestrous cycle is defined as the interval between two successive occurrences of oestrus. Thus, in koalas, it is important to distinguish between non-mated oestrous cycles $(32.9 \pm 1.1$ days) that do not contain a luteal phase and mated oestrous cycles that do (52.5 \pm 0.8 days). However, as the duration of the follicular phase in a species showing induced ovulation varies according to the time during oestrus when mating occurs, a more precise definition of the duration of the oestrous cycle is the period from commencement of the luteal phase (mating) to day 1 of the subsequent oestrus. In koalas, this period is $49.5 \pm 1.0$ days. As most marsupials ovulate spontaneously, comparisons of the duration of the oestrous cycle in koalas with that of other marsupials need to be based on estimates of the oestrous cycle that incorporate the luteal phase. On this basis, the oestrous cycle of the koala is exceptionally long, and is exceeded only by that of the kowari (Dasyuroides byrnei) and Matschie's tree kangaroo (Dendrolagus matschiei) which have oestrous cycles of 60 and 63 days, respectively (Fletcher, 1989; Flannery et al., 1996).

Typically, progestogen profiles of pregnancy and the luteal phase of the non-pregnant oestrous cycle in marsupials are not significantly different in duration or magnitude (Tyndale-Biscoe and Renfree, 1987). Preliminary evidence presented in the present study indicates that this axiom of marsupial reproductive biology may not hold true for the koala. Progestogen concentrations throughout pregnancy were found to be significantly higher than those of the luteal phase of mated but non-parturient koalas. However, this comparison was based on only six pregnant and six mated but non-parturient koalas, thus further observations are required before the possibility of maternal recognition of pregnancy or a supplementary endocrine role for the koala embryo or extra-embryonic membranes can be validated. In addition, it was unclear whether absence of pregnancy in the mated but non-parturient females was associated with fertilization failure or embryonic loss. Further studies involving the use of a vasectomized male to induce a sterile mated cycle would help to clarify the difference between progestogen concentrations of pregnant and mated but nonpregnant koalas.

Results from the present study indicate that in koalas 
gestation (34.8 days) occupies $70 \%$ of the oestrous cycle (49.5 days) and that parturition typically occurs before termination of the luteal phase. As a mating-induced luteal phase has yet to be demonstrated convincingly in any other marsupial, and in light of the reproductive characteristics outlined above, the koala does not fit into any of the four reproductive patterns proposed by Tyndale-Biscoe and Renfree (1987). Hence, there is a strong argument for classifying the koala in a reproductive group of its own.

The present study demonstrates that oestrous behaviours in koalas, such as jerking, pseudo-male mounting, urinating, bellowing and restlessness, are all closely associated with increasing or high concentrations of plasma oestradiol and, therefore, could be considered genuine displays of behavioural oestrus. Other studies (Smith, 1980; Blanshard, 1994; Johnston, 1994) did not identify urination as a distinctive oestrous behaviour, although Smith (1980) did note that both the male and female urinated after copulation.

The duration of oestrus reported in the present study was similar to that described by Thompson (1987) and Johnston (1994), but substantially greater than the $2.5-24 \mathrm{~h}$ reported by Smith (1980). The difference in the duration of oestrus is probably related to the mixed sex enclosures used by Smith (1980) compared with the separate sex housing system used in the present study (Blanshard, 1994). In the study of Smith (1980), females that were not separated from males would be expected to have little time to display oestrus before a male attempted copulation. In the present study, females were 'teased' each day without direct physical contact with a male, so that oestrus was allowed to run its full course.

Smith (1980) reported that oestrous behaviours in the female koala ceased the day after a successful copulation, a finding that was confirmed by the present study. The shortening of behavioural oestrus after mating is further secondary evidence that, in the koala, ovulation and the resulting luteal phase are induced by mating. Observations of a rapid cessation of oestrous behaviour after mating were generally coincident with a decrease in oestradiol concentration. However, the importance of decreasing oestradiol concentrations to the cessation of oestrus is still equivocal, as the later stages of oestrus during non-mated cycles did not appear to require the support of continuing high oestradiol concentrations.

In the present study, females that displayed pseudo-male mounting behaviour usually chose to mount females who where also in oestrus. This finding indicates that, as is observed in cows and other mammals, female koalas can recognize other oestrous females, either by their behaviour or pheromonally. The functional significance of oestrous behaviour in the koalas studied here is unclear, and it may be that it is an artefact of captivity. Although oestrus may be difficult to detect in the field, it is surprising that there are no records of oestrous behaviours in wild koalas. Whether or not such behaviour is an artefact of captivity, the expression of oestrous behaviour in the captive female koala makes the selection of female koalas for artificial insemination a relatively straightforward procedure.

Results from the present study indicate that pregnancy in the koala can occur if the female is mated up to 7 days after the onset of oestrus. Behavioural oestrus in one female koala in this study lasted up to 19 days, and it would be useful to know whether induction of ovulation is still possible after this time and more importantly whether the ovulated oocyte is capable of being fertilized. Such a period of time might represent a substantial window of opportunity in which the female could locate and mate with a male. In combination with the facility of induced ovulation, prolonged oestrus may confer an important adaptation for breeding success in an essentially solitary animal.

Characterization of the oestrous cycle and possible mechanism of ovulation in the koala, as described in this study, forms the basis of the information required to develop an artificial insemination protocol in this species. In addition, knowledge gained from an improved understanding of the fundamental reproductive physiology and behaviour of the koala will also be important in the development of conservation management plans, either for improving or reducing reproductive potential.

The authors would like to thank Tom Sakurabu, the current General Manager of Lone Pine Koala Sanctuary (LKPS), and Toshi Akiyama (former General Manager) for allowing access to their facilities and collaboration with their staff. In particular, Jacque Brumm and Karen Neilson who helped in oestrous detection procedures and collecting blood samples. The authors would also like to acknowledge the generous support of Jim Kelly of Bioquest Ltd (North Ryde, Sydney) for providing the laboratory space and equipment to run hormone assays. The authors are grateful to $\mathrm{F}$. N. Carrick for providing a critical review of the manuscript. The work presented in this paper was supported by research grants supplied by Lone Pine Koala Sanctuary and the University of Queensland.

\section{References}

Banks DR (1986) Physiology and endocrinology of the feline oestrous cycle. In Current Therapy in Theriogenology pp 795-800 Ed. DA Marrow. WB Saunders Company, Philadelphia

Blanshard W (1994) Medicine and husbandry of koalas. In Wildlife, Proceedings Number 223 pp 547-623. University of Sydney Post Graduate Committee in Veterinary Science, Sydney

Bravo PW, Fowler ME, Stabenfeldt GH and Lasley BL (1990) Ovarian dynamics in the llama Biology of Reproduction 43 579-585

Briese D (1970) The Oestrous Cycle of the Koala (Phascolarctos cinereus) Honours Research Project, Department of Zoology, The University of Adelaide, Adelaide

Brown AS (1987) Infertility in the Female Koala PhD Thesis, The University of Queensland, Brisbane

Crawford JL, Shackell GH, Thompson EG, McRead BJ and Hurst PR (1997) Pre-ovulatory follicle development and ovulation in the brushtail possum (Trichosurus vulpecula) monitored by repeated laparoscopy Journal of Reproduction and Fertility 110 361-370

Curlewis JD, Axelson M and Stone GM (1985) Identification of the major steroids in ovarian and adrenal venous plasma of the brushtail possum (Trichosurus vulpecula) and changes in the peripheral level of oestradiol and progesterone during the reproductive cycle Journal of Endocrinology 105 $53-62$

Fadem BH and Rayve R (1985) Characteristics of the oestrous cycle and influence of social factors in grey short-tailed opossums (Monodelphis domestica) Journal of Reproduction and Fertility 73 337-342

Feldman DS and Gagnon J (1991) Stat View Student Manual for the Mactintosh Abacus Concepts, Berkeley, CA

Flannery TF, Martin R and Szalay A (1996) Tree Kangaroos: A Curious Natural History Reed Books, Port Melbourne

Fletcher TP (1989) Plasma progesterone and body weight in the pregnant and non-pregnant Kowari, Dasyuroides byrnie (Marsupialia: Dasyuridae) Reproduction Fertility and Development 1 65-74 
Godfrey GK (1975) A study of oestrus and fecundity in a laboratory colony of mouse opossums (Marmosa robinsonii) Journal of Zoology, London 175 541-555 Hammond MA (1925) Reproduction in the Rabbit Oliver and Boyd, London

Handasyde KA (1986) Factors Affecting the Reproduction in the Female Koala Phascolarctos cinereus, PhD Thesis, Monash University, Victoria

Handasyde KA, McDonald IR, Than KA, Michaelides J and Martin RW (1990) Reproductive hormones in the koala. In Biology of the Koala pp 203-210 Eds AK Lee, KA Handasyde and GD Sanson. Surrey Beatty \& Sons Pty Ltd, Chipping North, New South Wales

Harder JD and Fleming MW (1981) Estradiol and progesterone profiles indicate a lack of endocrine recognition of pregnancy in the opossum Science 212 1400-1402

Harder JD, Hinds LA, Horn CA and Tyndale-Biscoe CH (1985) Effects of removal in late pregnancy of the corpus luteum, Graafian follicle or ovaries on plasma progesterone, oestradiol, LH, parturition and post-partum oestrus in the tammar, Macropus eugenii. Journal of Reproduction and Fertility 75 449-459

Hartman CG (1923) The oestrous cycle in the opossum American Journal of Anatomy 32 353-421

Herbert F (1972) Behavioural patterns. In Reproduction in Mammals, Book 4, Reproductive Patterns pp 34-68 Eds CR Austin and RV Short. Cambridge University Press, London

Hinds LA and Smith MJ (1992) Evidence from plasma progesterone concentrations for male-induced ovulation in an Australian marsupial, the brush-tailed bettong, Bettongia penicillata. Journal of Reproduction and Fertility 95 291-302

Hinds LA, Fletcher TP and Rodger JC (1996) Hormones of oestrus and ovulation and their manipulation in marsupials Reproduction, Fertility and Development 8 661-672

Johnston SD (1994) The Reproductive Biology of the Queensland Koala Honours Thesis, Department of Zoology, The University of Queensland, Brisbane

Johnston SD (1999) Studies Towards the Development of an Artificial Insemination Program in the Koala (Phascolarctos cinereus) PhD Thesis, School of Veterinary Science and Animal Production and Department of Zoology, The University of Queensland, Brisbane

Johnston SD, McGowan MR, O'Callaghan PO, Cox R and Nicolson V (2000) Natural and artificial methods of inducing the luteal phase in the koala (Phascolarctos cinereus) Journal of Reproduction and Fertility 120 59-64
Martinez-Esteve P (1937) Le cycle sexual vaginal chez le marsupial Didelphys azarae. Comptes Rendus de Societe de Biologie, Paris 124 502-504

Musa B, Sieme H, Merkt H, Hago BED, Cooper MJ, Allen WR and Jöchle W (1993) Manipulation of reproductive functions in male and female camels Animal Reproduction Science 33 289-306

O'Callaghan P (1996) Growth and mortality of koala pouch and back young Proceedings of the Australian Koala Foundation Annual Conference pp 101-109

Perret M and Ben M'Barek S (1991) Male influence on the oestrous cycles in the female woolly opossum (Caluromys philander) Journal of Reproduction and Fertility $\mathbf{9 1} 557-566$

Ramirez VD and Lin Soufi W (1994) The neuroendocrine control of the rabbit ovarian cycle. In The Physiology of Reproduction pp 585-611 Eds E Knobil and JD Neill. Raven Press, New York

Sharman GB and Calaby JH (1964) Reproductive behaviour in the red kangaroo, Megaleia rufa, in captivity CSIRO Wildlife Research 958-86

Shaw G and Renfree MB (1984) Concentrations of oestradiol-17 $\beta$ in plasma and corpora lutea throughout pregnancy in the tammar wallaby, Macropus eugenii. Journal of Reproduction and Fertility 72 29-37

Short R (1982) Oestrous and menstrual cycles. In Reproduction in Mammals, Book 3, Hormonal Control of Reproduction pp 115-152 Eds CR Austin and RV Short. Cambridge University Press, Sydney

Smith M (1980) Behaviour of the koala, Phascolarctos cinereus (Goldfuss) in captivity V. Sexual behaviour Australian Wildlife Research 7 41-51

Smith MJ (1992) Evidence from the oestrous cycle for male induced ovulation in the brush-tailed bettong, Bettongia penicillata (Marsupialia: Potoroidae) Journal of Reproduction and Fertility 95 283-289

Smith MJ (1994) Male induced oestrus and ovulation in female brush-tailed bettongs (Bettongia penicillata) suckling a young in the pouch Reproduction, Fertility and Development 6 445-449

Thompson VD (1987) Parturition and development in the Queensland koala Phascolarctos cinereus adustus at San Diego Zoo International Zoo Yearbook 26 217-222

Tyndale-Biscoe CH and Renfree M (1987) Monographs on Marsupial Biology, Reproductive Physiology of Marsupials University of Cambridge Press, Cambridge 